\title{
Optimal Sizing of Cylindrical Receivers for Surround Heliostat Fields using FluxTracer
}

\author{
Victor Grigoriev \\ Energy, Environment and \\ Water Research Centre (EEWRC) \\ The Cyprus Institute \\ Athalassa Campus \\ 20 Konstantinou Kavafi Street \\ Aglantzia, Nicosia 2121, Cyprus \\ Email: v.grigoriev@cyi.ac.cy
}

\author{
Marios Constantinou \\ Energy, Environment and \\ Water Research Centre (EEWRC) \\ The Cyprus Institute \\ Athalassa Campus \\ 20 Konstantinou Kavafi Street \\ Aglantzia, Nicosia 2121, Cyprus \\ Email: m.constantinou@cyi.ac.cy
}

\author{
Kypros Milidonis \\ Energy, Environment and \\ Water Research Centre (EEWRC) \\ The Cyprus Institute \\ Athalassa Campus \\ 20 Konstantinou Kavafi Street \\ Aglantzia, Nicosia 2121, Cyprus \\ Email:k.milidonis@cyi.ac.cy
}

\section{Clotilde Corsi}

Research School of Electrical, Energy

and Materials Engineering,

The Australian National University,

Canberra, Australia

Email: clo.corsi@gmail.com

\author{
Manuel Blanco* \\ Energy, Environment and \\ Water Research Centre (EEWRC) \\ The Cyprus Institute \\ Athalassa Campus \\ 20 Konstantinou Kavafi Street \\ Aglantzia, Nicosia 2121, Cyprus \\ Email:m.blanco@cyi.ac.cy
}

\begin{abstract}
This article presents an innovative approach for optimizing the dimensions of cylindrical receivers for solar tower systems. In this approach, a single set of rays, representative of a complete annual ray-tracing simulation of the solar tower system, is used and processed to evaluate numerous receiver designs simultaneously and to select the optimum. The simultaneous evaluation of receiver designs is achieved by exploiting the geometrical properties of the intersection between a ray and a cylinder, which allows estimating the annual energy intersected by receiver of different heights without the need for processing the annual set of rays more than one time. Once the annual intersected energy is known for each receiver, the application of a costing function estimating the receiver cost as a function of its surface area allows to estimate their cost and, therefore, to select the receiver dimensions that will yield a minimum surface area for a given annual energy interception factor. The overall workflow to carry out the mentioned receiver optimization approach has been implemented by adapting several open source tools The Cyprus Institute (CyI) is developing in collaboration with the Australian National University (ANU) to assist in the modelling, analysis, design and optimization of Concentrated Solar Thermal (CST) systems. This article also presents a detailed overview of the overall simulation workflow as well as a case study demonstrating the capabilities of the approach.
\end{abstract}

${ }^{*}$ Corresponding author 


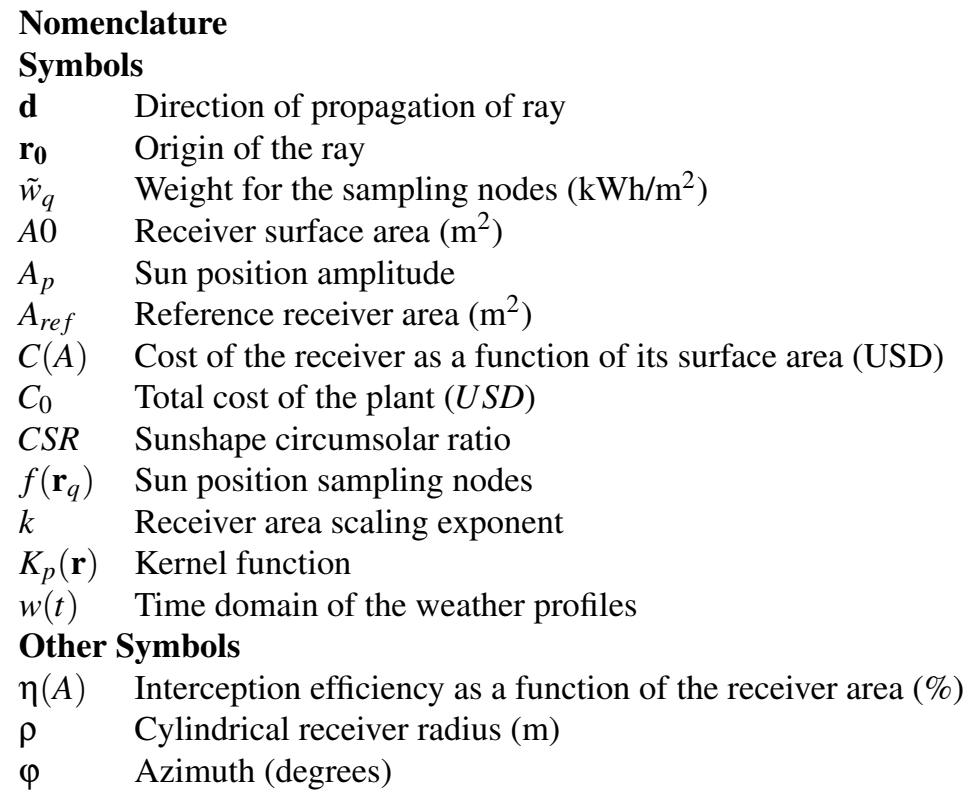

\section{Introduction}

The majority of large commercial solar tower plants being deployed in the world have two common characteristics: they have large surrounding heliostat fields, with a maximum heliostat-to-receiver distance often exceeding $1 \mathrm{~km}$, and cylindrical vertical receivers $(1 ; 2)$. While designing these plants, the optimization of the receiver dimensions is crucial, since the cost of the receiver increases monotonically with increasing surface area, and typically amounts to around 10-17\% of the total capital investment cost of the power plant (3). Moreover, the receiver's efficiency significantly affects the performance of the plant, and the amount of the energy produced. Because of this, most the computational tools to assist in the design and simulation of solar tower systems provide the option of optimizing the receiver's geometry. Many of these tools are discussed elsewhere in the literature $(4 ; 5 ; 6 ; 7 ; 8)$. In general, the most common approach to optimize the dimensions of a cylindrical receiver using any of those tools entails:

- Modelling the influence of the receiver's geometry (height and diameter) on the economic performance of the plant, as measured by one or several quantitative economic indicators, such as the Levelized Cost of Energy (LCOE) by considering the influence of the receiver geometry parameters on the annual energy yield of the plant and associate revenues, as well as on the annual capital and Operation and Maintenance (O\&M) costs.

- Finding the values of the receiver's geometry parameters that maximize the plant's economic performance by running the energy and cost models repeatedly using an appropriate optimization algorithm.

This approach can be implemented with the assistance of classical tools such as DELSOL3 (9) or more modern tools such as SolTrace (10), Tonatiuh (11), HFLCAL (12), Heliosim (13), SoFiA (14), SAM (15), SOLSTICE (16), SolarTherm (17), SolarPILOT (8), SunFlower (18), Tracer (19), etc. Some of these tools use cone-optics, while others use Monte Carlo ray-tracing (MCRT) to simulate the optical behavior of the heliostat field and its interaction with the receiver. Some of them are capable of carrying out the complete classical approach without the need for scripts or for a person-in-the-loop, while others require scripting or other type of coding and adaptation. Finally, some of them such as Tonatiuh (11) are quite flexible regarding the type of heliostat tracking that can be simulated, while others such as SolarPILOT (8), can only handle heliostats with azimuth-elevation tracking systems. Furthermore, some of the mentioned tools can be used to estimate the annual output and final LCOE of a solar thermal electric power plant, while others are able only to estimate the radiant concentrated solar energy delivered by the heliostat field to the receiver for a specified sun position, and therefore require either the use of additional tools to estimate the annual electricity yield of the plant or the re-formulation of the receiver geometry optimization problems in terms of the energy delivered by the heliostat field to the receiver. 
Typically, the computation of the annual energy yield of the solar tower plant requires modelling the behavior of the plant for a series of instances of time, i.e. sun positions and atmospheric conditions, which are appropriately selected to estimate the annual yield accurately with low or minimum computational effort.

Drawbacks of this common approach to optimize the geometry of cylindrical receivers are: factor matrix of the second stage into a a new matrix that represents the variation of the user-defined metric with receiver dimensions, and finds the dimensions of the receiver which optimizes the metric.

The following subsections describe these three different stages in detail, emphasizing how the proposed approach is implementing them in a modular and flexible way, which enhances the options available to the user regarding how to tailor the execution of the approach for the specificity of the problem to be analyzed. Thus, emphasizing how this new approach addresses all the drawbacks of the current common approach.

\subsection{Stage 1: Generation of rays}

- It is either too monolithic or too ad-hoc. In those cases in which the program used implements the complete optimization approach, the user is limited to select only the few options for system optimization that are available. In those cases in which the program is not used to implement the complete approach, ad-hoc solutions must be developed for solving specific problems.

- If ray-tracing is used to simulate the optical behavior of the heliostat field, the same rays have to be generated over and over again to evaluate different receiver configurations at identical instants in time or they have to be stored and processed over and over.

In relation to the second point mentioned above, efforts have been devoted to reduce the computational effort and execution time of ray tracing simulations, especially for large heliostat fields. For example, in STRAL(20) instead of casting the rays from a virtual "solar" surface above the reflector field, the rays are generated directly on the surface of the reflectors, eliminating the processing of rays from the sun that will miss the reflecting surfaces. This strategy, along with the CPU parallelization of the code, results in a significantly reduced execution time. A similar approach for generating rays is used in the sbpRAY (21) ray tracer, but, to further reduce the execution time of the code this is massively parallelized using GPU's. Among ray tracing tools that are also execution-time efficient in calculating large field simulations are SOLSTICE (16) and Aiosim (13) in which the sampling of the rays are also performed on the first intersection with the primary reflector surfaces. and stochastic optimization algorithms (22). In this approach, while numerous geometrical configurations are considered for the reflector field and receiver, the stochastic optimization seeks to identify and progress to intensive ray tracing only those configurations that are promising within the population. Candidate configurations that are under-performing are early discarded by performing MCRT simulations using a relatively lower number of rays.

The approach to optimize the dimensions of cylindrical receivers presented in this article is based on a one-time postprocessing of all the rays generated during the simulation of a solar tower system to estimate its annual energy yield. As such, it addresses the drawbacks of the common approach mentioned above, while it can take advantage of any advances in MCRT programs (present and future) as long as the MCRT programs make it possible to export their rays for further post-processing.

\section{The new approach}

In the proposed approach, the optical behavior of a heliostat field for a given location is appropriately captured for a complete year in the form of a large set of rays. Once obtained, this set of rays is efficiently post-processed to estimate how the annual energy interception factor of a cylindrical receiver varies as a function of diameter and height. This information is then combined with a model that connects the size of the receiver with whatever metrics the user wants to optimize, and he optimum size of the receiver is found in a very computationally efficient way.

The overall approach, is composed of three distinct stages. The first stage, which is the most computationally intensive, generates a set of rays that appropriately captures the overall annual energy behavior of the heliostat field. In the second stage, a two-dimensional matrix representing the variation of the annual intercept factor of the cylindrical receiver as a function of its height and diameter is generated, by appropriately post-processing just one-time the set of rays generated in the first stage. The annual intercept factor is the fraction of the annual energy send by the heliostat field towards the receiver which is actually intercepted by the receiver. Finally, the third stage utilizes a model correlating the receiver dimensions with 2.1.1 Sampling of sun positions and weights

The annual performance of the power plant depends on the weather data. This data is usually provided as a time-series, such as 'typical meteorological year' (TMY) file, with a fixed time step. However, running the MCRT simulation for each different sun position is computationally expensive and needs a large amount of data storage. To reduce the number of 
ray-tracing simulations needed to estimate the annual performance of the plant, the motion of the sun in the spatial domain can be discretized and effective weights for the selected positions of the sun computed (23).

In the approach proposed in this paper, this is performed using an open source computational tool called SunPath. This tool, which is being developed by The Cyprus Institute (CYI), takes as input the geographical location of the plant (latitude and longitude), the desired angular resolution for sky partitioning, and the location's weather data, or a clear sky model. It returns the sun positions and the values of the effective weights (i.e. a list of points that includes the azimuth, elevation and weight) to be used in the ray tracing simulations to achieve an accurate estimate of the annual energy sent by the heliostat field to the receiver, and the annual energies intercepted and delivered by it. The Graphical User Interface (GUI) of SunPath is shown in Fig. 1.

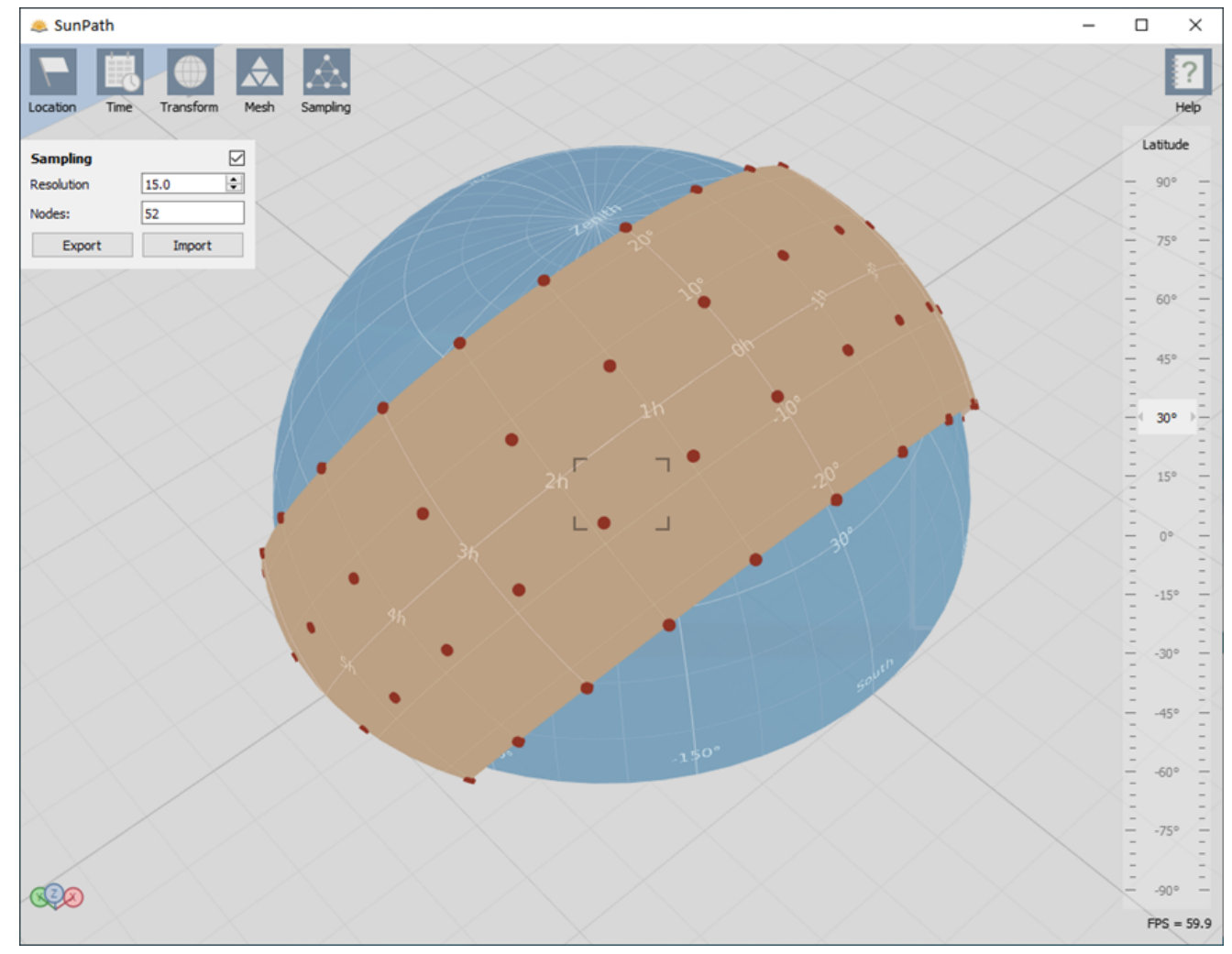

Fig. 1. The Graphical User Interface (GUI) of SunPath

In SunPath, the position of the sun is described in terms of declination and hour angle. The sampling points are obtained by taking an equidistant grid and stretching it to fit within the boundaries of the annual sun path. Any function which depends on position of the sun is represented as a sum over nodes

$$
f(\mathbf{r})=\sum_{p} A_{p} K_{p}(\mathbf{r})
$$

where $A_{p}$ are the amplitudes, and $K_{p}(\mathbf{r})$ are the kernel function of the nodes defined as

$$
K_{p}(\mathbf{r})=\exp \left(\frac{\mathbf{r} \cdot \mathbf{r}_{p}-1}{\sigma^{2}}\right)
$$

Although the choice of kernel is somewhat arbitrary, the radial Gaussian distribution gives rather accurate results for interpolation. If the function is known at the sampling points $f\left(\mathbf{r}_{q}\right)$

$$
f\left(\mathbf{r}_{q}\right)=\sum_{p} A_{p} K_{p}\left(\mathbf{r}_{q}\right),
$$


then the amplitudes $A_{p}$ can be found by solving the linear matrix equation

$$
A_{p}=\sum_{q} K_{p q}^{-1} f_{q}
$$

\subsection{Stage 2: Generation of the annual intersect factor matrix}

The annual intercept factor matrix is a two-dimensional matrix representing the variation of the annual intersect factor of the cylindrical receiver as a function of its height and diameter. It is calculated using FluxTracer (25), a MCRT post-

The kernels can also be used to compute the overlap of the nodes with weather profiles in time domain $w(t)$. This simplifies the calculation of annual integrals in the following form

$$
\int_{\text {year }} f(t) w(t) d t=\sum_{p} A_{p} \int_{\text {year }} K_{p}[\mathbf{r}(t)] w(t) d t .
$$

And after substitution of Eqn. (4)

$$
\int_{\text {year }} f(t) w(t) d t=\sum_{q} \tilde{w}_{q} f_{q}
$$

where $\tilde{w}_{q}$ the weights of the nodes are given by

$$
\tilde{w}_{q}=\sum_{p} K_{p q}^{-1} \int_{y e a r} K_{p}(t) w(t) d t .
$$

For the proposed approach, the optimization workflow incorporates Tonatiuh as the tool for performing the ray tracing simulations. In general, the flux calculations in Tonatiuh are performed in terms of photons, with a ray representing the path followed by a photon while interacting with the surfaces of the solar concentrating system. The power intercepted by a surface is equal to the number of intercepted photons multiplied by the power assigned to each photon. The latter quantity is calculated in Tonatiuh based on the DNI, the area of the projection of the scene in a plane perpendicular to the sunrays and the number of rays cast. In the case of performing annual simulations based on the sun-sampling approach, the ray tracing is performed for the weighted set of sun positions as described above.

The weights $\tilde{w}_{q}$ are calculated based on the DNI data, and in this article, for simplicity, a clear-sky model was applied (24). The weights have units of $\mathrm{kWh} / \mathrm{m}^{2}$, and can be interpreted as an average DNI (in $\mathrm{kW} / \mathrm{m}^{2}$ ) for a selected sun position multiplied by the time (in hours) spent by the sun in the vicinity of this position. These weights are then used instead of DNI in Tonatiuh, and as output they give the energy of photons for annual simulations. The annual energy intercepted by the receiver is equal then to the energy of intercepted photons.

In summary, at this stage of the optimization workflow, SunPath produces a list of annual representative sun-sampling points which contains the elevation, azimuth and the corresponding weight in $\mathrm{kWh} / \mathrm{m}^{2}$ for each one of the points.

\subsubsection{Ray-tracing}

The output list of sun-sampling points obtained during the sun-sampling stage is then fed into Tonatiuh, which is responsible for performing the ray-tracing simulations for each point of the annual sun-sampling list. The sun-sampling list is loaded into the scripting editor of Tonatiuh, in which a dedicated script takes over and automatically performs the annual simulation point by point. At this stage of the optimization workflow, Tonatiuh outputs the annual set of rays, which combines all the rays obtained for each simulation point for the heliostat field being investigated. Within Tonatiuh, the different segments that define a ray can be identified based on their interceptions with the surfaces of the solar concentrating system. For this case, the ray segments that are saved for post-processing are those that are reflected by the heliostat field and reach a "virtual" plane that is placed at an appropriate distance over the focal point as shown in Fig. 2. Virtual here means that the plane's optical properties are treated so as to allow the rays to pass freely from the light source surface (i.e. the sun) to the heliostat field without changing any of their characteristics, but the reflected rays form the heliostat to the virtual plane are stopped on the latter. The absence of a receiver in the ray-tracing simulations and the addition of the virtual plane, allows obtaining rays from Tonatiuh which are independent of the receiver size and dimensions. Of course, all the heliostats are set to aim at the focal point of the heliostat field, usually located along the vertical center axis of the receiver.

processing tool designed to aid in the analysis, design, and optimization of solar concentrators and receiver systems. As with SunPath, the development of FluxTracer as an open source software tool is being led by CyI 


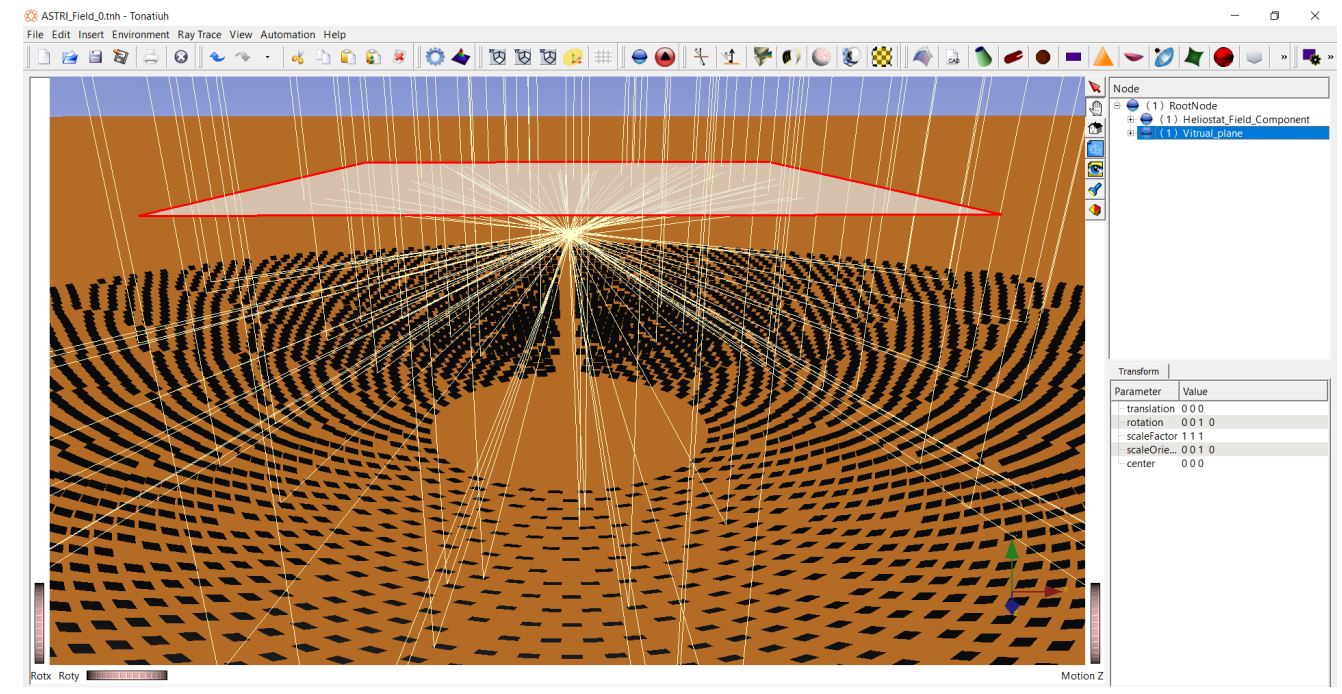

Fig. 2. The use of a virtual plane instead of a receiver in Tonatiuh. Rays pass freely from the "sun" surface to the heliostat field without changing any of their characteristics.

The annual set of rays reflected by the heliostat field towards the receiver, generated at the ray tracing stage, is postprocessed by FluxTracer using the 'cylindrical receiver analysis' functionality of the program to generate the annual intersect factor matrix. This matrix is then fed to the next stage of the optimization workflow. To generate the annual intersect factor matrix, the user of FluxTracer, should provide the following information to the program:

- The interval (dMin, dMax) of diameters of the cylindrical receivers to be analyzed,

- The interval (hMin, hmax) of heights of the cylindrical receivers to be analyzed.

- The number of divisions of the interval of diamaters (dDivs) to be considered.

- The number of divisions of the interval of heights (hDivs) to be considered.

- The coordinates of the focal point of the optical system of the plant, which is usually located along the center axis of the receiver. (These coordinates should be identical to the coordinates of the focal point defined in the ray-tracing software.)

The coordinates of the focal point of the optical system of the plant to be provide to FluxTracer is usually located along the center axis of the receiver. These coordinates should be identical to the coordinates of the focal point defined in the ray-tracing software.

To build the annual intersect factor matrix FluxTracer computes the intersection points of each one of the rays in the annual set of rays with the cylindrical surface of the receiver. A point on the cylindrical surface of a receiver can be described by the following equation:

$$
\mathbf{r}=\rho \cos \varphi \hat{\mathbf{x}}+\rho \sin \varphi \hat{\mathbf{y}}+z \hat{\mathbf{z}}
$$

where the radius $\rho$, azimuth $\varphi$ and height $\mathrm{z}$ are the cylindrical coordinates. A point on a ray can be described as:

$$
\mathbf{r}=\mathbf{r}_{\mathbf{0}}+t \mathbf{d}
$$

where $\mathbf{r}_{0}$ is the origin, $\mathbf{d}$ is the direction of propagation, and $t$ is the distance from the origin. The intersection points of cylinder and ray, therefore, satisfy the following equation:

$$
\rho^{2}=\left(r_{0 x}+t d_{x}\right)^{2}+\left(r_{0 y}+t d_{y}\right)^{2}
$$

As a quadratic in $t$, Eqn. (10) can be rewritten as: 


$$
\left(d_{x}^{2}+d_{y}^{2}\right) t^{2}+2\left(r_{0 x} d_{x}+r_{0 y} d_{y}\right)+\left(r_{0 x}^{2}+r_{0 y}^{2}-\rho^{2}\right)=0
$$

In general, there can be two roots that satisfy the above equation. The smaller root $t_{1}$ corresponds to the first intersection with the surface. The height of the intersection point is:

$$
z=r_{0 z}+t_{1} d_{z}
$$

The root should be within the length of the ray segment $0 \leq t_{1} \leq t_{\max }$. The parameter $t_{\max }$ is specific to the implementation obtained as segments connecting the photons. The intersections with the virtual surface are also stored, and $t_{\max }$ depends on the altitude of the surface. Therefore, it is important to set the surface sufficiently high over the focal point. Otherwise, the ray is excluded from consideration. The case when $d_{x}^{2}+d_{y}^{2}=0$ corresponds to rays which are parallel to the cylindrical surface and thus are neglected.

The functionality within FluxTracer investigates the design space by using cylinders of fixed diameter and explores all combinations of the set cylinder heights due to the fact that if a ray intersects a smaller in height cylinder, it will always intersect a larger in height cylinder of the same diameter. However, if a ray intersects a smaller in diameter cylinder, it does not necessarily intersect a larger in diameter cylinder of the same height as illustrated in Figure 3.

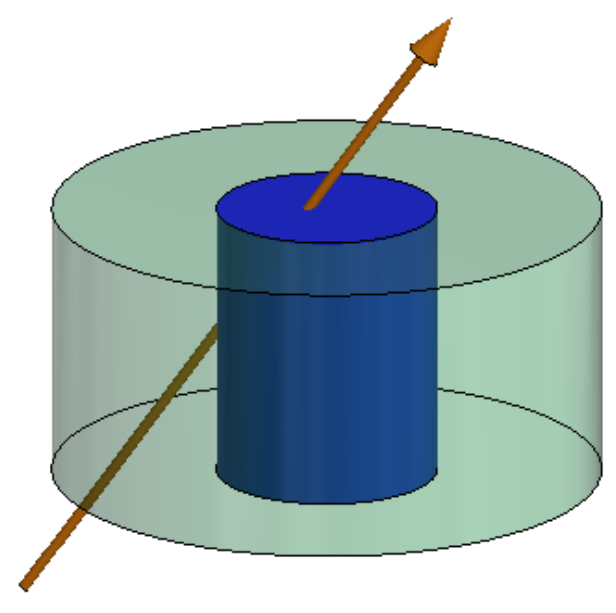

(a)

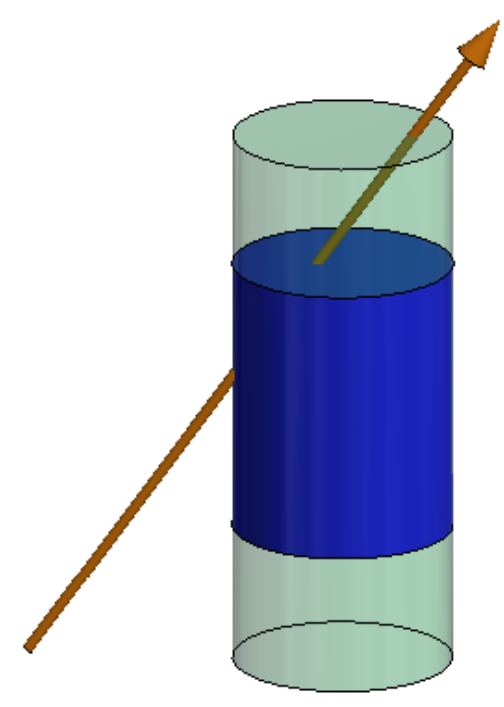

(b)

Fig. 3. Intersection of a ray with cylinder, (a) Cylinders of different diameters and of same height: if a ray intersects a smaller in diameter cylinder, it does not necessarily intersect a larger in diameter cylinder of the same height, (b) cylinders of different height and of same diameter: if a ray intersects the smaller in height cylinder, it will always intersect a larger in height cylinder of the same diameter

Based on the above, to build the two dimensional (hDivs, dDivs) annual intersect factor matrix, FluxTracer only needs to process the rays in the annual set of rays dDivs times, and not hDivs $\mathrm{x}$ dDivs times, which typically is a significant reduction in the computational effort.

\subsection{Stage 3: Generation of annual final metrics matrix}

In the last stage of the proposed approach, the previously generated annual intercept factor matrix is related to the metric or metrics that the user is interested in optimizing, for example, LCOE. The results are then processed to represent the variation of the user-defined metric according to the intersect factor matrix and finally, are translated into a user-friendly form that is useful for the interpretation of the results. For implementing the last stage of the proposed approach, any scientific 
technical computing and visualization tool can be employed in the workflow, such as the open-source ParaView (26) or the commercial software Mathematica.

For the purpose of demonstrating the proposed approach, which addresses the size optimization of cylindrical receivers, the annual intercept factor matrix is related to the cost of the receiver $C(A)$ as a function of its surface area $A$. The metric used is defined by the following function, which is directly proportional to the electricity price, usually measured in cents of Euros or Dollars per kWh:

$$
\frac{C_{0}+C(A)}{\eta(A)}
$$

while $\eta(A)$ is the interception as a function of the receiver area and $C_{0}$ the total cost of the plant excluding the cost of the receiver. The optimal size (i.e surface area) of the receiver is then selected based on the minimum of this function. The cost of a receiver can be estimated using the following power-law approximation (8), (13):

$$
C=C_{r e f}\left(\frac{A}{A_{\text {ref }}}\right)^{k}
$$

where $C_{r e f}$ is the cost per receiver reference area to account for receiver installation costs, including labor and equipment, $A_{r e f}$ is the receiver area on which the receiver reference cost is based, and $k$ is a scaling exponent that defines the nonlinear relationship between receiver cost and receiver area based on the reference cost conditions provided. The reference values suggested in the SAM software (15) are $C_{r e f}=104.6 \mathrm{M} \mathrm{USD}, A_{r e f}=1571 \mathrm{~m}^{2}$, and $k=0.7$.

The overall proposed approach is implemented with the assistance of the scientific workflow software DataWolf, whose active development as an open-source software project is led by the National Center for Supercomputing Applications (NCSA) of the University of Illinois. DataWolf can be used to integrate, combine and execute a series of software tools through a sequence of connected steps, ensuring seamless interconnection between the tools, and assuring the exchange of information through input and output files of specified formats. Figure 4 illustrates the overall workflow of the framework implemented for the proposed approach.

\section{Analysis of the ASTRI reference plant}

To illustrate the use of the proposed cylindrical receiver size optimization approach, the heliostat field of the solar tower defined as reference plant within the Australian Solar Thermal Research Initiative (ASTRI) was chosen as a test case, since published data exists regarding its main techno-economic specifications (27), (1).

As mentioned before, the overall optimization approach is implemented using the open-source scientific workflow system DataWolf. A screenshot of the workflow as implemented within DataWolf is shown in Fig. 5. The workflow does not show any optimization loop because the optimization of the cylindrical receiver geometry is carried out by FluxTracer. After calculating the optimal receiver size through the proposed approach, the results are compared to the techno-economic specifications of the actual ASTRI reference field.

\subsection{ASTRI reference plant heliostat field layout}

The ASTRI reference plant is a typical two tank molten salt solar tower plant with a gross capacity of $111 \mathrm{MWe}$, a net capacity of $100 \mathrm{MWe}$, four hours of thermal storage and solar multiple of 1.8, which is located in Alice Springs (latitude: $-23.8^{\circ}$ North, longitude: $133.88^{\circ}$ East, elevation: $547 \mathrm{~m}$ ). This heliostat field, shown in Fig. 6 , is of a surrounded type and it is composed of 6,177 mirrors of $37.21 \mathrm{~m}^{2}(6.1 \mathrm{~m} \times 6.1 \mathrm{~m})$ each, with a total reflecting area of $222,846 \mathrm{~m}^{2}$.

Initially, the reference power plant of ASTRI was designed for $100 \mathrm{MWe}$ of net capacity and had a receiver of $15 \mathrm{~m}$ in diameter and $18.67 \mathrm{~m}$ in height. Later on, the heliostat field layout for a $25 \mathrm{MWe}$ ASTRI reference plant was generated based on the initial 100 MWe heliostat field layout by scaling down four times the original layout, i.e., by dividing the dimensions of the original layout by two on the direction of each axis. In the latter case, the receiver had a diameter of $7.5 \mathrm{~m}$ and a height of $9.33 \mathrm{~m}$. The $25 \mathrm{MWe}$ ASTRI reference field layout is the one considered in this paper.

\subsection{Sun sampling for Alice Springs}

To execute the first stage of the optimization workflow, the sampling points over Alice Springs were obtained using SunPath as explained in 2.1.1. The input to SunPath were the geographical location of Alice Springs, the desired angular 


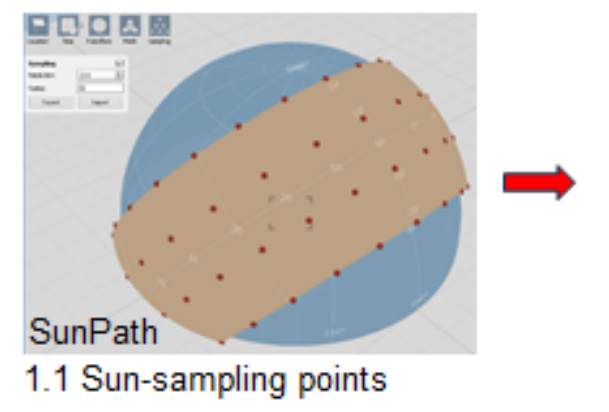

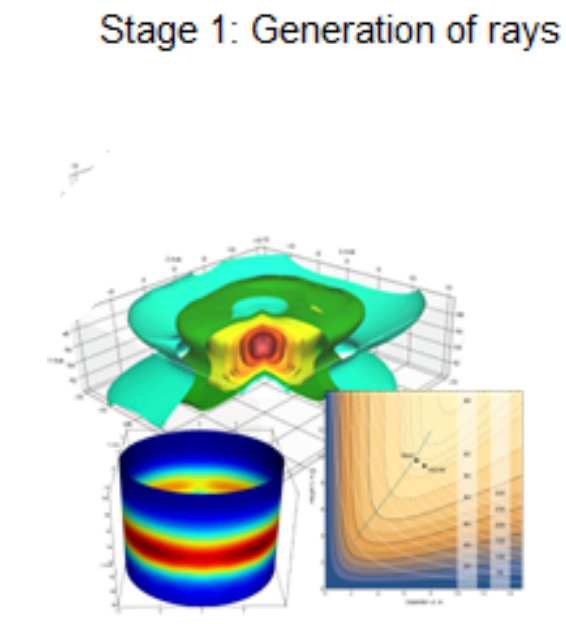

Stage 3: Annual final metrics matrix
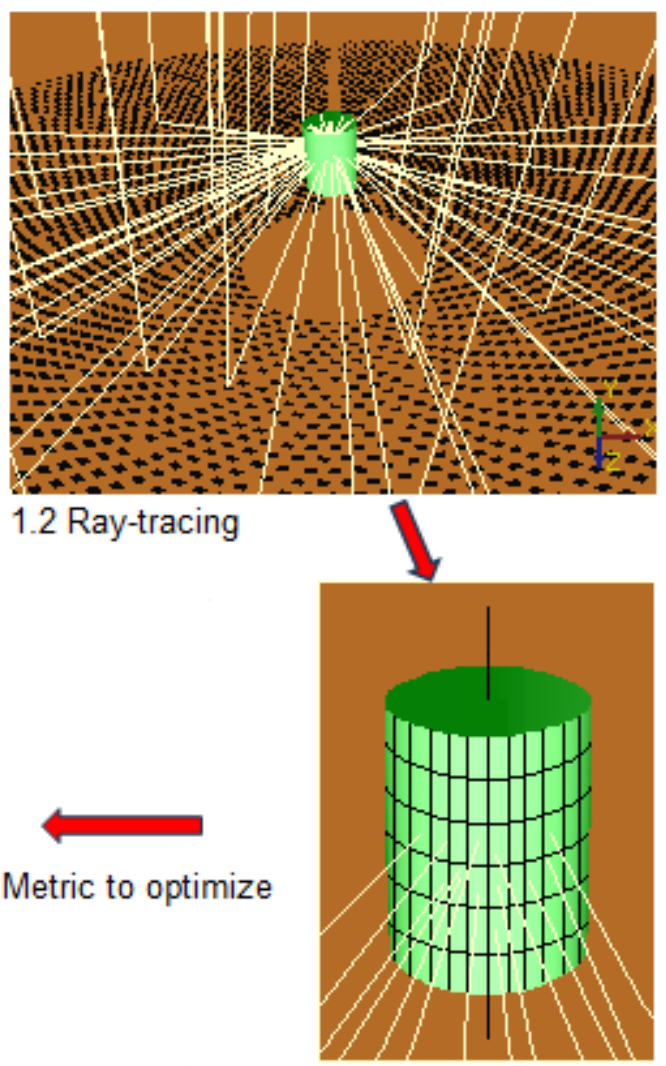

Stage 2: Annual intersect factor
matrix calculation

Fig. 4. Proposed simulation workflow of the proposed approach

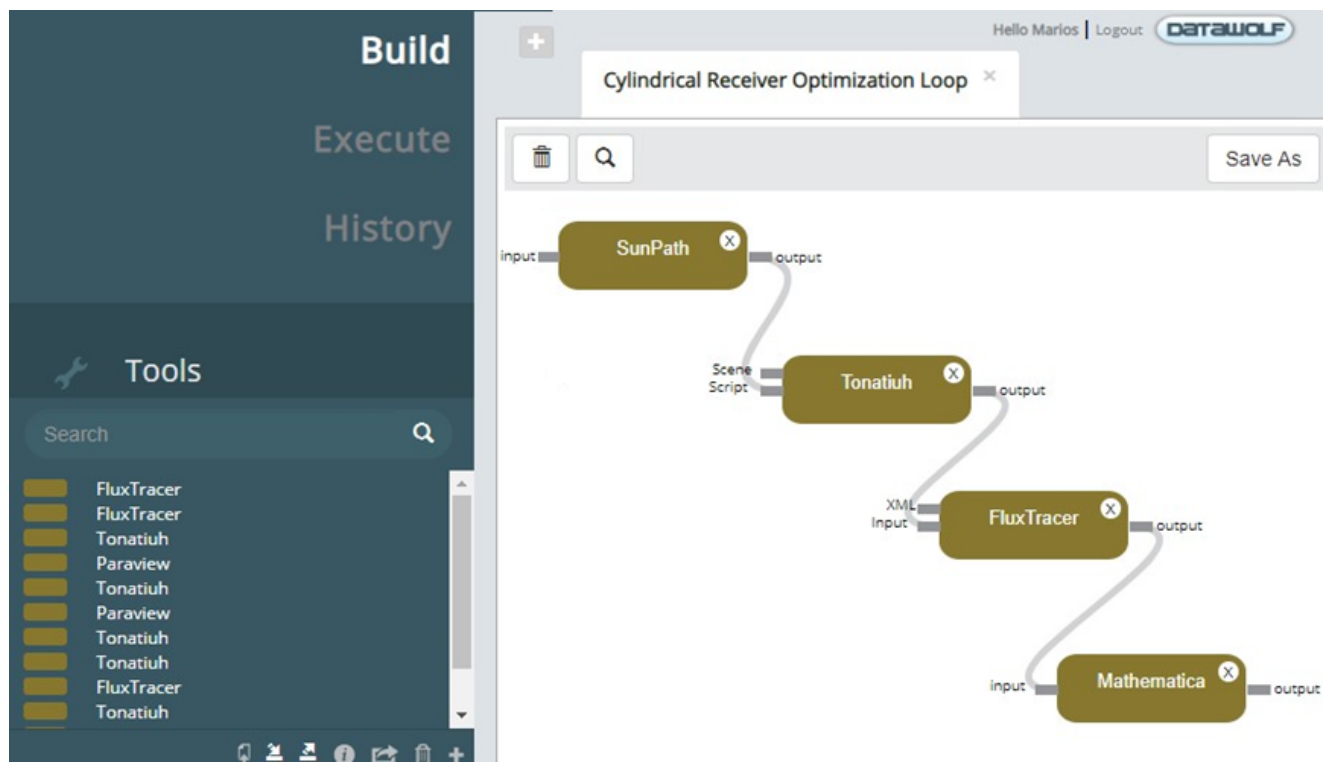

Fig. 5. The optimization workflow as implemented in DataWolf system

resolution for partitioning of the sky and the clear sky model to consider. As output, SunPath provided the sun positions and their corresponding values of the effective DNI weights in the form of a list.

For the present study, the grid of sun positions adopted for the ASTRI annual simulations is shown in Fig. 7 and corresponds to an angular resolution of 15.6 degrees, thus 50 simulation points are considered. 


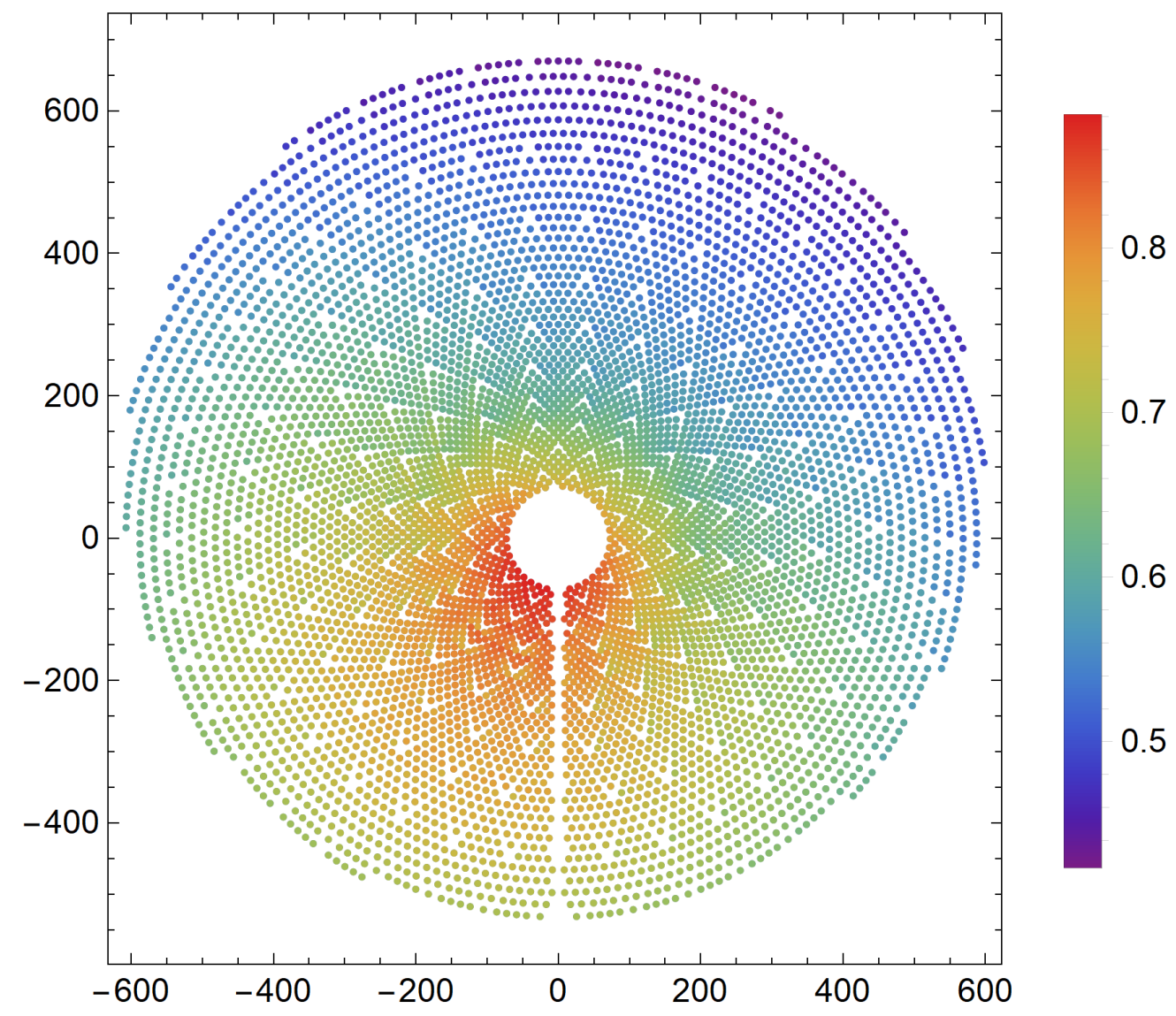

Fig. 6. Top view of the ASTRI heliostat field colored by annual efficiency

\subsection{Ray-tracing simulations}

To execute this phase of the optimization workflow, Tonatiuh ray-tracer was fed with:

- The list of 50 sun-sampling points previously obtained for representing the annual sun path over Alice Springs, which is used as input parameters in the ray tracing simulations for generating the required annual set of rays. Within Tonatiuh, this list is loaded and each case simulated using the program's embedded script interpreter.

- The Tonatiuh scene file, which contains the layout of the ASTRI heliostat field, the heliostat aiming position data and tracking configuration and all the necessary information regarding the characteristics of the reflecting surfaces.

For each one of the sun-sampling points, the entire heliostat field of the ASTRI reference plant was simulated in Tonatiuh, with each heliostat aiming at the focal point. As discussed in Section 2.1.2, the scene includes no receiver component but instead includes a virtual surface (a horizontal plane) above the focal point. This virtual plane was placed parallel to the ground and centered along the vertical axis, $20 \mathrm{~m}$ above the focal point, ensuring that the rays reflected from the heliostats towards the aiming point will cross the focal region of the heliostat field (Fig. 2). The reference center for the configuration $(0,0,0) \mathrm{m}$ is placed at the center of the tower base and the aiming point coordinate is at $(0,95.0,0) \mathrm{m}$.

In the details of the simulation, the sunshape selected was the Buie sunshape (28), (29) with a circumsolar ratio (CSR) of $2 \%$. The reflectivity of the heliostats was set to $100 \%$ and their slope error distribution was set to normal with a standard deviation of $1.53 \mathrm{mrad}$. For the ray-tracing simulations, 300 million rays were cast over the entire heliostat field resulting in many millions of rays traversing the region of interest.

At this stage of the optimization process, the output of Tonatiuh simulations are ASCII binary ray data files storing the intersection related information of each ray intersecting the heliostats and the virtual plane of the scene. In sequence, these binary files are fed and read by FluxTracer, which then computes the annual intersection matrix using the cylindrical receiver analysis functionality as described in Section 2.2. Separately, an XML configuration file is provided to FluxTracer which contains other optimization parameters, namely the minimum and maximum allowable diameters and heights for the receiver.

The intercept factor matrix generated by FluxTracer for this case is a two-dimensional matrix in CSV format, representing the variation of the annual intercept factor of the cylindrical receiver as a function of its height and diameter. 


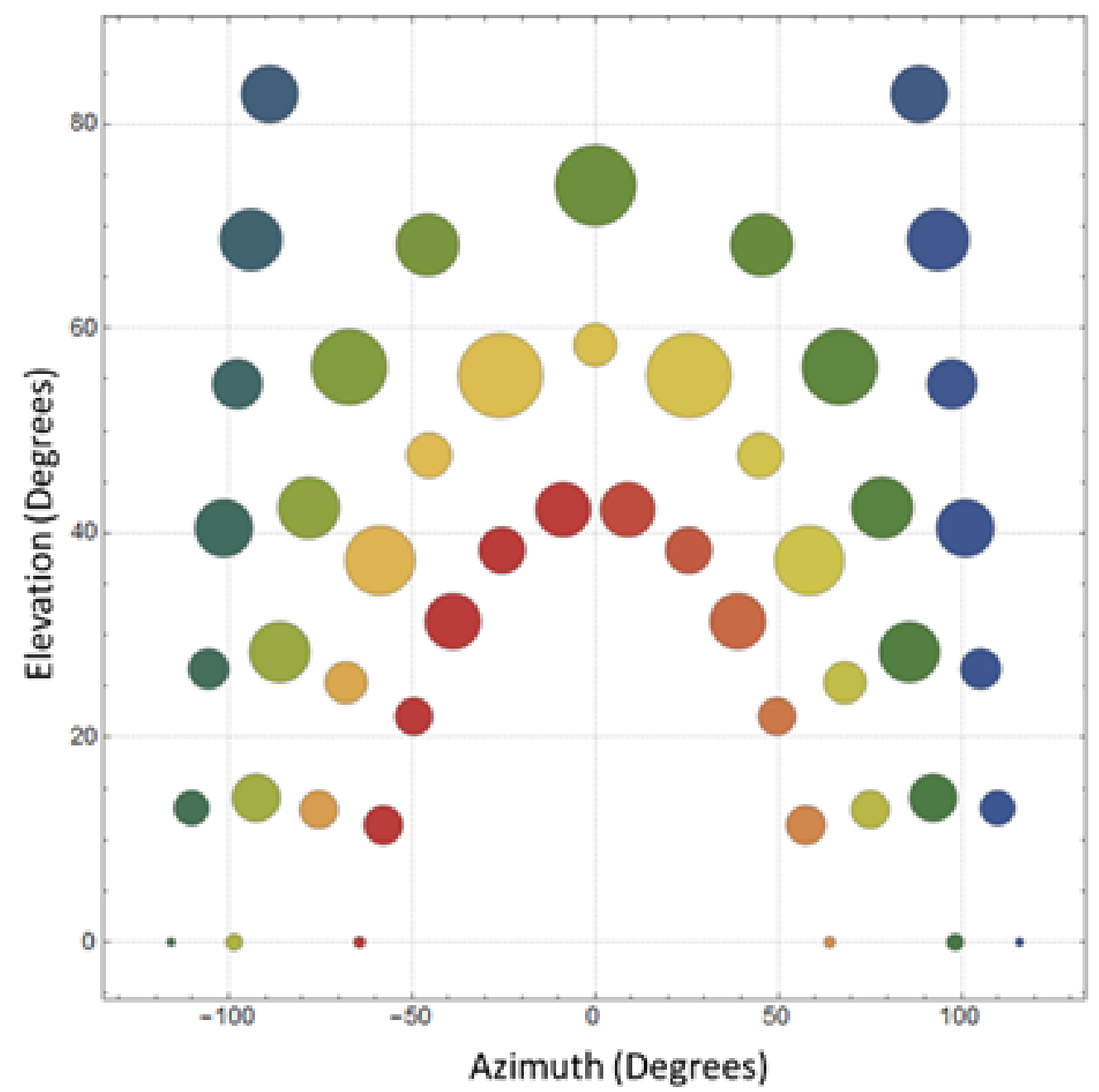

Fig. 7. Schematic of annual sun path adopted for the ASTRI simulations. The bubble size corresponds to the weight used in $\mathrm{kWh} / \mathrm{m}^{2}$, while the coloring represents the declination angle.

Finally, the two-dimensional annual intercept factor matrix computed by FluxTracer is loaded into Mathematica, where a script is used to calculate the costing metric as discussed in section 2.3 and to generate the final annual metrics matrix. In the same script, figures are plotted to allow evaluation of the results.

\section{Results and Discussion}

As mentioned before, the final stage of the cylindrical receiver optimization includes the implementation of the relationship between the annual intersect factor matrix to the receiver costing function that was introduced earlier in Eqn. (13) and calculation of the annual final metric matrix.

The most straightforward way to visualize the latter, is by constructing a 2D contour map as shown in Fig. 8, which correlates the receiver's height at the vertical axis against its diameter on the horizontal axis for a given intersection percentage, shown in black lines. The white lines lying on top of the contours correspond to a constant receiver area, while the blue line corresponds to the maximal intersection percentage for the given receiver area. The receiver area shown ranges between 25 and $300 \mathrm{~m}^{2}$.

The first thing that can be noticed is that the intercept factor for a fixed diameter is a monotonic function of height, while, the intercept factor for a fixed height is not a monotonic function of diameter. This is expected since the rays intersecting the cap surfaces of the receiver do not contribute to the number of rays with valid receiver intersection.

Figure 9 shows a plot of the normalized price of electricity function with regards to the receiver area, based on Eqn. (13. The script calculates the global minimum of this function. Then, it finds the optimum receiver area for the given heliostat field. The dashed horizontal line in the figure corresponds to the minimum of the function, and its intersection point (labeled 'FTOpt') with the first vertical line corresponds to the optimum receiver area calculated by the optimization process. This 


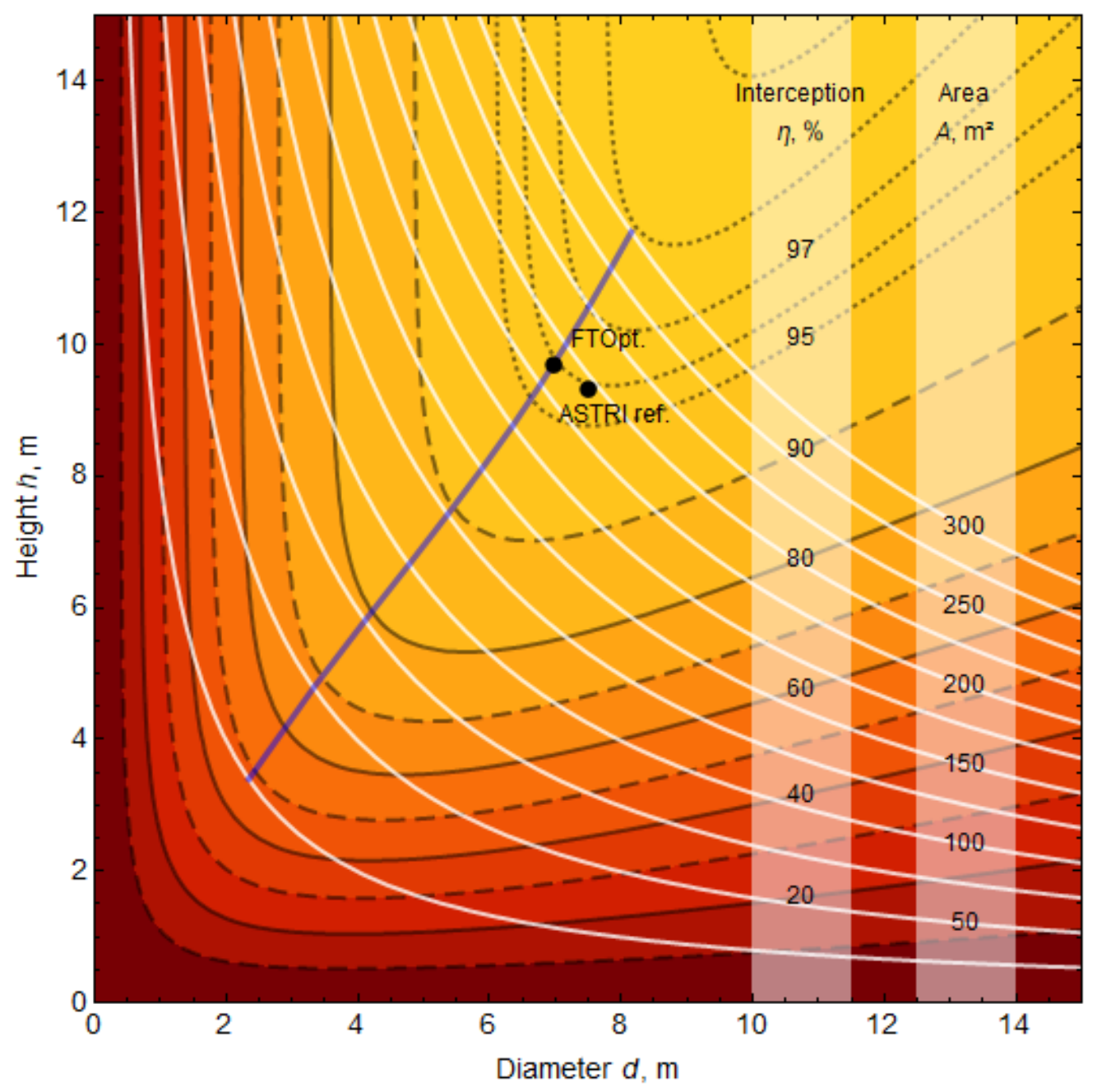

Fig. 8. ASTRI reference plant receiver intercept factor and receiver area, as a function of diameter and height

optimum receiver area is $210.7 \mathrm{~m}^{2}$. The actual receiver considered in the ASTRI reference plant has a diameter of $7.5 \mathrm{~m}$ and a height of $9.33 \mathrm{~m}(1)$ and, therefore, an area of $219.95 \mathrm{~m}^{2}$.

According to the SAM model (Eqn. (14), the cost of the ASTRI reference plant receiver is estimated to be $C=$ 26.41 MUSD. The cost of the receiver contributed to $12 \%$ of the total construction cost of the power plant $C_{t o t}$. Thus, $C_{t o t}=C / 0.12=220.08$ MUSD. The cost includes the erection of the heliostat field, the power block, the storage, the tower structure and so on. Thus, the total cost $C_{0}$ excluding the receiver can be estimated to be $C_{0}=C_{\text {tot }}-C_{r e f}=193.67$ MUSD.

For comparison, the point that corresponds to the actual receiver area of the ASTRI reference field is also shown in Fig. 9 (labeled 'ASTRI ref'). It is clear that although there is a global minimum in the function, it is very shallow. This means that the influence of the area of the receiver in the electricity price is relatively minor, e.g., an increase in the receiver area from $150 \mathrm{~m}^{2}$ to $300 \mathrm{~m}^{2}$ will result in an increase of the electricity price of only $1 \%$.

The two points 'FTOpt' and 'ASTRI ref' are also shown on Fig. 8. Taking into account the calculated optimum receiver area, the script positions the FTOpt point on the intersection between the corresponding constant area white line and the blue line that represents the maximal intersection percentage for the given receiver area.

It can be seen from the 'FTOpt' point in Fig. 8 that the height and diameter of the optimal receiver differs from the actual ASTRI diameter-height combination. It is clear that the size of the ASTRI reference plant receiver is slightly larger than required.

In terms of cost, the receiver oversize translates into an overspend of nearly 0.8 MUSD as seen in Fig. 10 which shows a plot of the receiver cost as a function of the receiver area, i.e. Eqn. (14).

A line plot of the interception percentage as a function of the receiver area is also shown in Fig. 11 along with the two points 'FTOpt' and 'ASTRI ref'. For the given heliostat field, although the actual receiver of the ASTRI reference plant achieves a slightly higher interception (96.24\%) in comparison to the interception of the optimum receiver identified by the proposed approach (95.92\%), the sizing of the ASTRI reference receiver is not optimum in terms of minimizing the 


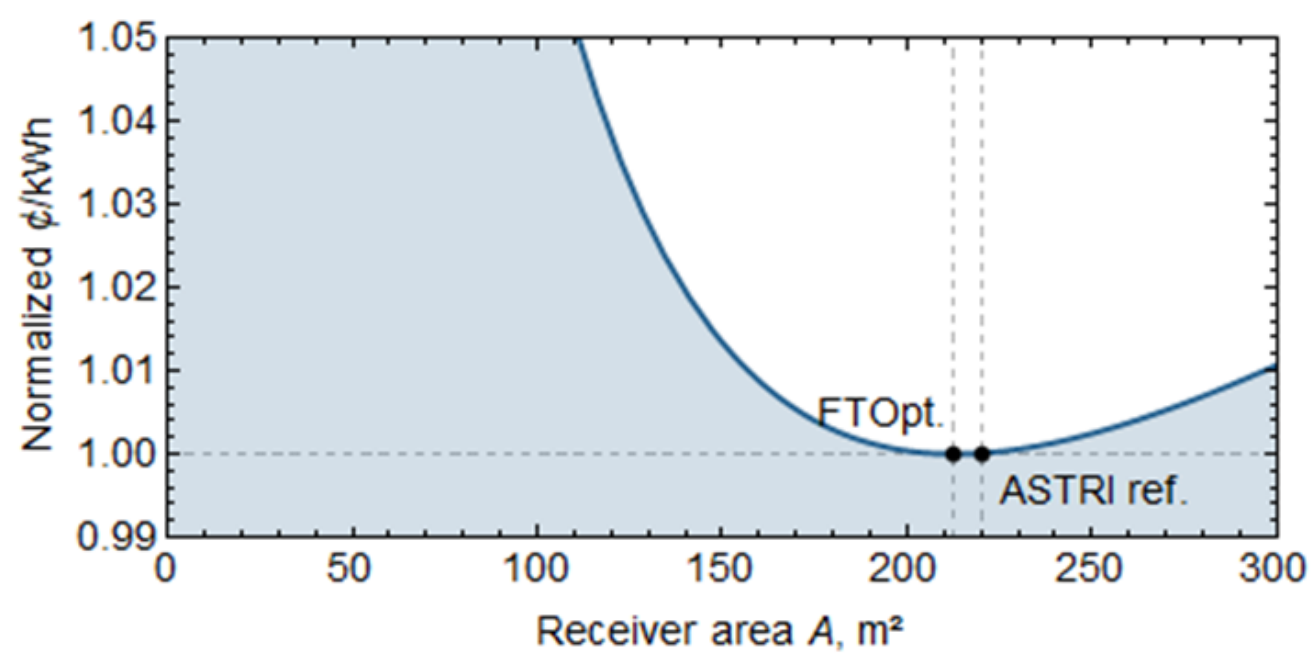

Fig. 9. Normalized price of electricity according to Eqn. (13) as a function of the receiver area. The points ASTRI ref. and FTOpt. correspond to the ASTRI reference plant and the results of FluxTracer optimization respectively.

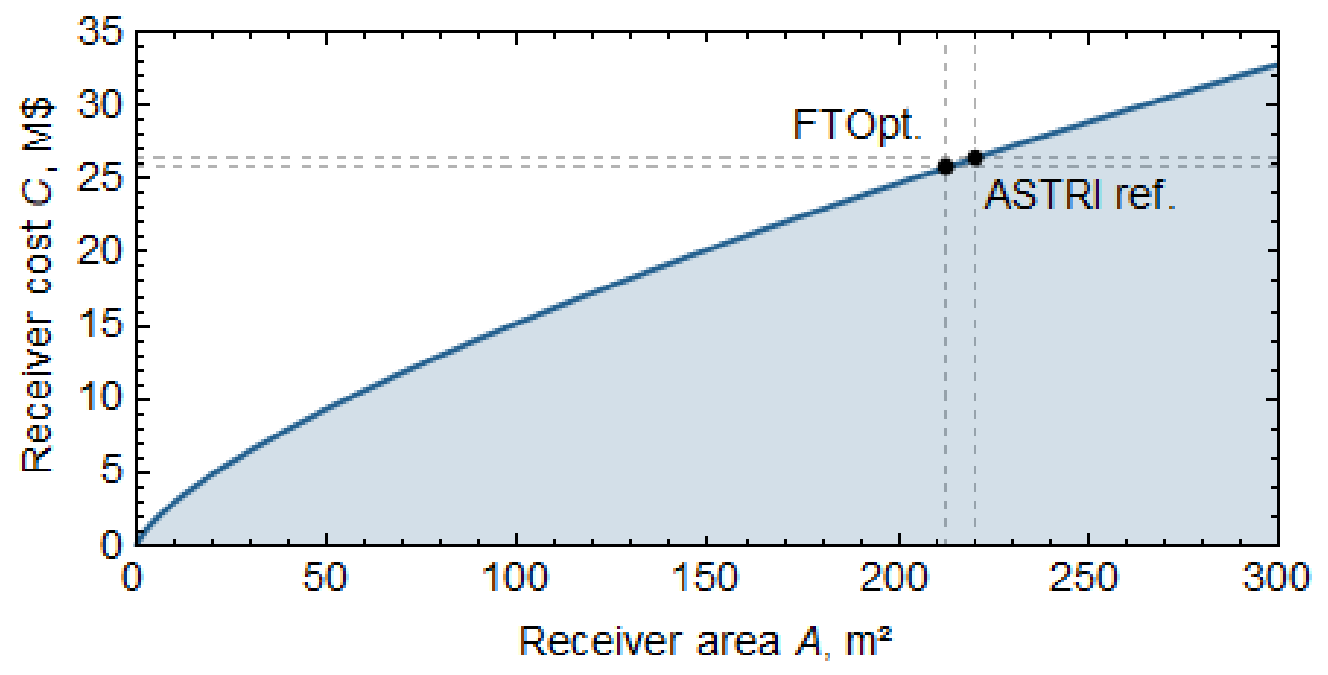

Fig. 10. Receiver cost as a function of its surface area

normalized price of electricity function, since the increase in cost associated to the increase in surface is not compensated by the increase in electricity production associated to the increase on the number of intercepted rays along the year. It is noted however that thermal losses are an important additional consideration in receiver design which were not included in the present calculation.

Table 1 summarizes the differences between characteristics of the optimal receiver found using the approach presented in this paper and the receiver of the 25 MWe ASTRI reference solar tower power plant.

\section{Conclusions}

A new innovative and modular approach for optimizing the size of external cylindrical receivers for surrounded heliostat fields has been developed. The approach is based on the combined use of three on-going open source computational tools, SunPath, Tonatiuh, and FluxTracer, while Mathematica was also used as a post-processing tool. The tools were linked and integrated using the DataWolf open-source scientific workflow framework.

The resulting approach was applied to the ASTRI reference heliostat field and the optimum receiver dimensions (height and diameter) were determined, with the figure of merit being a normalized price of electricity function similar to LCOE. It was shown that the optimization problem has a global minimum solution, but it is shallow and rather insensitive to the design parameters.

The novelty of the present approach to optimization of external cylindrical receivers is as follows: 


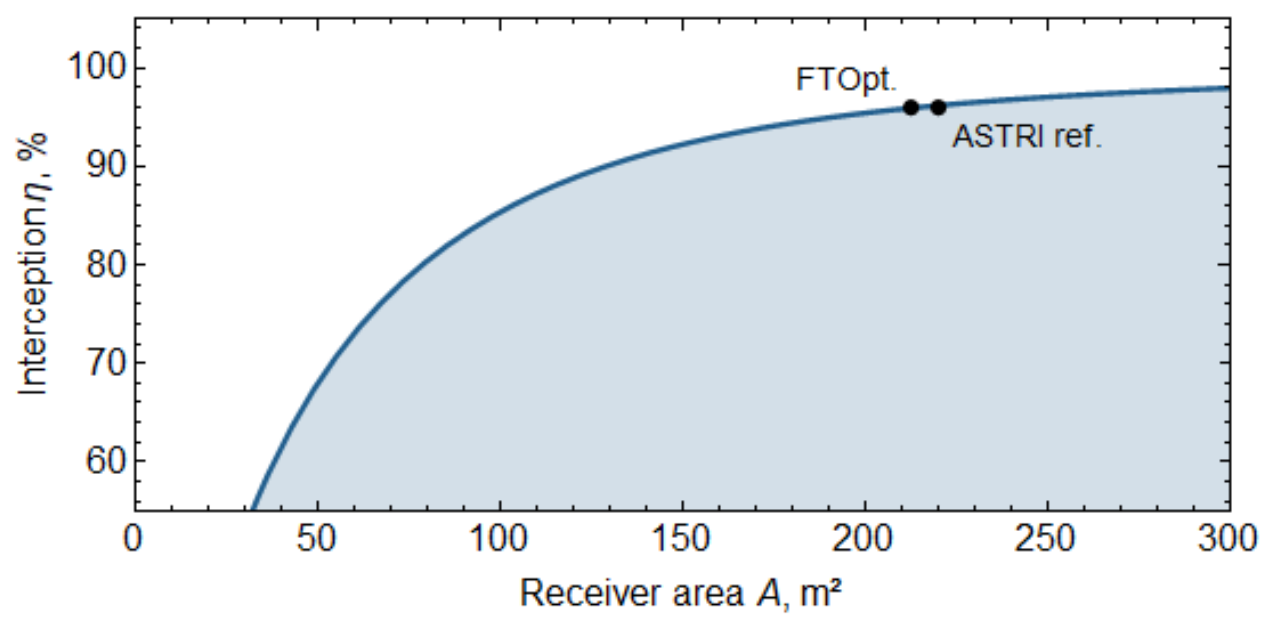

Fig. 11. The maximal interception as a function of receiver area

Table 1. Comparison of the actual ASTRI reference plant details to the ones calculated

\begin{tabular}{cll}
\hline Characteristic & ASTRI ref. & FTOpt. \\
\hline Diameter $(\mathrm{m})$ & 7.50 & 6.90 \\
Height $(\mathrm{m})$ & 9.33 & 9.71 \\
Area $\left(\mathrm{m}^{2}\right)$ & 219.95 & 210.70 \\
Interception (\%) & 96.24 & 95.92 \\
Cost (M USD) & 26.41 & 25.63 \\
\hline
\end{tabular}

- The cylindrical receiver geometry (height and diameter) can be optimized in a single run, without having to generate a new set of rays (i.e. run the ray-tracing software for each optimization iteration) for each different design. Instead, a single set of rays is used to calculate the optimum combination of height and diameter for the receiver centered at the user-defined point of interest, through post-processing analysis of the paths taken by those rays.

- The overall optimization process requires a low computing runtime. For example, given the ray-tracing data, for the ASTRI test case described in this paper, the optimization process took approximately two minutes on a HP Z4 G4 workstation computer equipped with Intel Xeon W-2133 processor / 6 cores, from which most of the runtime was actually used to read in the huge amount of ray-tracing data.

- The optimization approach is applicable to any type of heliostat tracking mechanism (for example, azimuth-elevation or tilt-roll), since FluxTracer post-processes data obtained from ray-tracing.

The tools and data needed for testing the optimization approach presented in this paper are available for downloading at the Confluence portal of the Energy Division of the Cyprus Institute used to host and foster the collaborative development of open-source computational tools for modeling, analysis, design and optimization of CST systems. The URL is https://scmt.cyi.ac.cy/confluence/.

\section{Acknowledgements}

This work is supported by the European Union's Horizon 2020 research and innovation programme within the context of the CySTEM ERA Chair project, under grant agreement No. 667942.

\section{References}

[1] Aghaeimeybodi, M. C., Beath, A. C., and Webby, B. U. o. S. A., 2017. Technoeconomic analysis of Solar Tower Reference plant. Tech. rep., CSIRO, Newcastle, NSW, Australia. 
[2] Ho, C. K. S. N. L., 2017. "Advances in central receivers for concentrating solar applications”. Solar Energy, 152, pp. 38-56.

[3] Rodríguez-Sánchez, M. R., Sánchez-González, A., Marugán-Cruz, C., and Santana, D., 2014. "New Designs of Moltensalt Tubular-receiver for Solar Power Tower". Energy Procedia, 49, pp. 504-513.

[4] Ho, C. K. S. N. L., 2008. "Software and Codes for Analysis of Concentrating Solar Power Technologies". Technical Report SAND2008-8053, p. 35.

[5] Garcia, P., Ferriere, A., and Bezian, J. J., 2008. "Codes for solar flux calculation dedicated to central receiver system applications: A comparative review". Solar Energy.

[6] Bode, S. J., and Gauché, P., 2012. "Review of optical software for use in concentrating solar power systems.”. In Proceedings of the Southern African Solar Energy Conference (SASEC 2012).

[7] Cruz, N. C., Redondo, J. L., Berenguel, M., Álvarez, J. D., and Ortigosa, P. M., 2017. "Review of software for optical analyzing and optimizing heliostat fields". Renewable and Sustainable Energy Reviews, 72, pp. 1001-1018.

[8] Wagner, M. J., and Wendelin, T., 2018. "SolarPILOT: A power tower solar field layout and characterization tool". Solar Energy, 171, pp. 185-196.

[9] Kistler, B. L. S. N. L., 1986. “A user's manual for DELSOL3: A computer code for calculating the optical performance and optimal system design for solar thermal central receiver plants". Technical Report SAND86-8018, p. 240.

[10] Wendelin, T., 2003. "Soltrace: A new optical modeling tool for concentrating solar optics". In International Solar Energy Conference.

[11] Blanco, M. J., Amieva, J. M., and Mancillas, A., 2005. "The Tonatiuh Software Development Project: An Open Source Approach to the Simulation of Solar Concentrating Systems”. In Computers and Information in Engineering, Vol. 2005, pp. 157-164.

[12] Schwarzbözl, P., Pitz-Paal, R., and Schmitz, M., 2009. "Visual HFLCAL - A Software Tool for Layout and Optimisation of Heliostat Fields". In SolarPACES 2009, T. Mancini and R. Pitz-Paal, eds.

[13] Potter, D. F., Kim, J.-S., Khassapov, A., Pascual, R., Hetherton, L., and Zhang, Z., 2018. "Heliosim: An integrated model for the optimisation and simulation of central receiver CSP facilities". AIP Conference Proceedings, 2033(1), 11, p. 210011.

[14] Gertig, C., Delgado, A., Hidalgo, C., and Ron, R., 2014. "SoFiA - A Novel Simulation Tool for Central Receiver Systems". Energy Procedia, 49, pp. 1361-1370.

[15] Blair, N. N., Dobo, A. P. N., Freeman, J. N., Neises, T. N., Wagner, M. N., Ferguson, T., Gilman, P., and Janzou, S., 2014. System Advisor Model, SAM 2014.1.14 : general description. NREL/TP ; 6A20-61019. National Renewable Energy Laboratory, Golden, CO.

[16] Caliot, C., Benoit, H., Guillot, E., Sans, J.-L., Ferriere, A., Flamant, G., Coustet, C., and Piaud, B., 2015. "Validation of a Monte Carlo Integral Formulation Applied to Solar Facility Simulations and Use of Sensitivities". Journal of Solar Energy Engineering, 137(2), 4, pp. 021019-1.

[17] Scott, P. A., Alonso, A. d. 1. C. C., Hinkley, J. T. C., and Pye, J. A., 2017. "SolarTherm: A flexible Modelica-based simulator for CSP systems". In AIP Conference Proceedings 1850. SOLARPACES 2016., AIP Publishing, pp. 1600261.

[18] Richter, P., Heiming, G., Lukas, N., and Frank, M., 2018. "SunFlower: A new solar tower simulation method for use in field layout optimization". In AIP Conference Proceedings 2033., p. 210015.

[19] Wang, Y., Potter, D., Asselineau, C.-A., Corsi, C., Wagner, M., Caliot, C., Piaud, B., Blanco, M., Kim, J.-S., and Pye, J., 2020. "Verification of optical modelling of sunshape and surface slope error for concentrating solar power systems". Solar Energy, 195, pp. 461-474.

[20] Belhomme, B., Pitz-Paal, R., Schwarzbözl, P., and Ulmer, S., 2009. "A new fast ray tracing tool for high-precision simulation of heliostat fields". Journal of Solar Energy Engineering, Transactions of the ASME, 131(3), pp. 03100210310028 .

[21] Gebreiter, D., Weinrebe, G., Wöhrbach, M., Arbes, F., Gross, F., and Landman, W., 2019. "SbpRAY - A fast and versatile tool for the simulation of large scale CSP plants". AIP Conference Proceedings, 2126(July).

[22] Asselineau, C.-A., Zapata, J., and Pye, J., 2015. "Integration of Monte-Carlo ray tracing with a stochastic optimisation method: application to the design of solar receiver geometry". Optics Express, 23(11), p. A437.

[23] Grigoriev, V., Corsi, C., and Blanco, M., 2016. "Fourier Sampling of Sun Path for Applications in Solar Energy.". AIP Conference Proceedings, 1734(1), 5, p. 1.

[24] Ineichen, P., 2008. "A broadband simplified version of the Solis clear sky model". Solar Energy, 82(8), pp. 758-762.

[25] Blanco, M., Constantinou, M., Corsi, C., Grigoriev, V., Milidonis, K., Panagiotou, C. F., Papanicolas, C. N., and Pye, J., 2019. "FluxTracer: A Ray Tracer Postprocessor to Assist in the Design and Optimization of Solar Concentrators and Receivers". Journal of Solar Energy Engineering, Transactions of the ASME.

[26] Ahrens, J. L. A., Geveci, Berk (Kitware, I., and Law, Charles (Kitware, I., 2005. "ParaView: An End-User Tool for 
[27] Coventry, J., Arjomandi, M., Barry, J., Blanco, M., Burgess, G., Campbell, J., Connor, P., Emes, M., Fairman, P., Farrant, D., Ghanadi, F., Grigoriev, V., Hall, C., Koltun, P., Lewis, D., Martin, S., Nathan, G., Pye, J., Qiu, A., Stuart, W., Tang, Y., Venn, F., and Yu, J., 2016. "Development of the ASTRI heliostat". p. 020005.

[28] Buie, D., Monger, A. G., and Dey, C. J., 2003. "Sunshape distributions for terrestrial solar simulations". Solar Energy, 74(2), pp. 113-122.

[29] Buie, D., Dey, C. J., and Bosi, S., 2003. "The effective size of the solar cone for solar concentrating systems". Solar Energy, 74(5), pp. 417-427. 